\title{
GROWTH FUNCTIONS FOR RESIDUALLY TORSION-FREE NILPOTENT GROUPS
}

\author{
ROGER C. ALPERIN AND BRIAN L. PETERSON
}

(Communicated by Warren J. Wong)

\begin{abstract}
We show that G. Baumslag's growth function for the free group is not algebraic.
\end{abstract}

Recently, G. Baumslag has introduced some new numerical invariants for the class $\mathscr{T}$ of finitely generated residually torsion-free nilpotent groups. Recall that for $n \geq 1$, we let $\gamma_{n+1}(X)=\left[\gamma_{n}(X), X\right], \gamma_{1}(X)=X$ and this defines the lower central series of the group $X$. Since the group $X / \gamma_{n+1}(X)$ is nilpotent it has a torsion subgroup consisting of all the elements of finite order; hence we can define the subgroup $\bar{\gamma}_{n}(X)$ of $X$ so that $\bar{\gamma}_{n}(X) / \gamma_{n+1}(X)$ is the torsion subgroup of the quotient group $\gamma_{n}(X) / \gamma_{n+1}(X)$. The class $\mathscr{T}$ can then be defined to consist of those groups which satisfy

$$
\bigcap_{n} \bar{\gamma}_{n}(X)=\{1\} \text {. }
$$

It follows easily from the definition of $\bar{\gamma}_{n}(X)$ and the finite generation of $X$ that groups in this class have the property that the successive quotients $\bar{\gamma}_{n}(X) / \bar{\gamma}_{n+1}(X)$ are finitely generated free Abelian groups; let the ranks of these finitely generated free Abelian groups be denoted $r_{n}(X)$. Let $s_{n}=\sum_{k=1}^{n} r_{k}$; the lower central series dimension of $X$ is

$$
\delta(X)=\lim \sup \left(\frac{\ln \left(s_{n}\right)}{\ln (n)}\right)
$$

and their growth series of $X$ is the generating function

$$
\operatorname{Gr}(X)=\sum_{n=1}^{\infty} s_{n}(X) z^{n}
$$

These invariants of $X$ are analogs of the notion of the dimension of a variety and the Poincare series of its associated graded local algebra. One would expect then that the size of $\delta(X)$, a measure of the degree of growth of the coefficients $s_{n}(X)$, is related to the nature of the function $\operatorname{Gr}(X)$, i.e. whether

Received by the editors May 31, 1989 and, in revised form, October 17, 1989.

1980 Mathematics Subject Classification (1985 Revision). Primary 20F18.

Key words and phrases. Residually torsion-free nilpotent groups. 
it is a polynomial, rational, algebraic or transcendental function. Baumslag has shown that (a) if $X \in \mathscr{T}$ is metabelian then $s_{n}$ is a polynomial in $n$ for all large $n$ and that $\delta$ is an integer and (b) if $X \in \mathscr{T}$ is Abelian by polycyclic then $\operatorname{Gr}(X)$ is a rational function [B]. He has asked if the growth series of the free group of rank $q$ is rational or not. We shall show that it is not algebraic.

The ranks of the quotients of the lower central series of a free group of rank $q, \mathscr{F}_{q}$, are well known [S, Chapter 4].

$$
r_{n}=\frac{1}{n} \sum_{d \mid n} \mu(d) q^{n / d}
$$

where $\mu(d)$ is the Moebius function. Rather than consider the series $\mathrm{Gr}$, we consider the series

$$
\operatorname{gr}(z)=\sum_{n=1}^{\infty} r_{n}(q) z^{n}
$$

Interchanging the order of summation we obtain

$$
\begin{aligned}
\operatorname{gr}(z) & =\sum_{n=1}^{\infty} \frac{1}{n} \sum_{d \mid n} \mu(d) q^{n / d} z^{n} \\
& =\sum_{d=1}^{\infty} \sum_{m=1}^{\infty} \frac{\mu(d)}{d m} q^{m} z^{d m} \\
& =\sum_{d=1}^{\infty} \frac{\mu(d)}{d} \sum_{m=1}^{\infty} \frac{\left(q z^{d}\right)^{m}}{m} \\
& =\sum_{d=1}^{\infty} \frac{-\mu(d)}{d} \ln \left(1-q z^{d}\right) .
\end{aligned}
$$

Formally, we can also obtain the derivative,

$$
\operatorname{gr}^{\prime}(z)=\sum_{d=1}^{\infty} \frac{\mu(d) q z^{d-1}}{1-q z^{d}} .
$$

Consider this function $\operatorname{gr}(z)$ as a function of the complex parameter $z$. We observe the following.

Lemma. If $w(z)$ is an algebraic function then it has at most a finite number of poles; its derivative $w^{\prime}(z)$ is also an algebraic function.

Proof. If $w$ satisfies a monic algebraic equation with coeffients which are rational functions then a solution can be found for all values of $z$ outside the finite set of poles of those coefficients; differentiation of the algebraic equation satisfied by $w$ gives an algebraic expression for $w^{\prime}$ in terms of $w$ and the conclusion follows.

Thus to show that $\operatorname{gr}(z)$ is not algebraic it suffices to show that its derivative has an infinite number of poles. Of course this is in some sense obvious since the poles occur at $q^{-1 / d}$ for square-free values of $d$. However we need to 
verify that this is in fact a valid series for $\operatorname{gr}^{\prime}(z)$ and that the poles do not somehow cancel out.

Proposition. The function $\operatorname{gr}^{\prime}(z)$ is not algebraic and hence neither is $\operatorname{gr}(z)$.

Proof. An easy comparision with the geometric series shows that the function $\operatorname{gr}^{\prime}(z)$ converges uniformly for $|z|<1 / q$ and hence that $\operatorname{gr}(z)$ converges uniformly there also. Moreover, if we consider $\operatorname{gr}^{\prime}(z)$ on any compact subset of $|z|<1$ it is easily seen to be meromorphic there since we need only delete a finite number of terms from its series expression above to get a holomorphic series. Thus we shall show that $\operatorname{gr}(z)$ is not algebraic by showing that $\operatorname{gr}^{\prime}(z)$ has simple poles at the values $q^{-1 / d}$ for square-free values of $d$, i.e., an infinite number of poles. Since the series for $\operatorname{gr}^{\prime}(z)$ converges absolutely in a deleted neighborhood of some particular $z_{0}=q^{-1 / d}$ we evaluate:

$$
\lim _{z \rightarrow z_{0}}\left(z-q^{1 / d}\right) \operatorname{gr}^{\prime}(z)=\lim _{z \rightarrow z_{0}}\left(z-q^{-1 / d}\right) \frac{\mu(d) q z^{d-1}}{1-q z^{d}} \neq 0
$$

and the conclusion now follows.

\section{REFERENCES}

[B] G. Baumslag, Some reflections on finitely generated metabelian groups, Contemp. Math. (to appear).

[S] J.-P. Serre, Lie algebras and Lie groups, Benjamin, New York, 1965.

Department of Mathematics and Computer Science, San Jose State University, San Jose, CALIFORNIA 95192 